\title{
ArcheoSciences
}

Revue d'archéométrie

\section{Amphores vinaires Gauloise 4 de Narbonnaise (France) exportées à Carthagène (Espagne) ? Analyse statistique exploratoire multidimensionnelle de données géochimiques}

Wine amphore Gauloise 4 from Narbonnensis (France) exported to Cartagena

(Spain)? Multidimensional statistical analysis of geochemical data

Thomas Delbey, Alejandro Quevedo et Fanette Laubenheimer

\section{(2) OpenEdition}

Journals

Édition électronique

URL : https://journals.openedition.org/archeosciences/4488

DOI : $10.4000 /$ archeosciences. 4488

ISBN : 978-2-7535-4778-0

ISSN : 2104-3728

Éditeur

Presses universitaires de Rennes

Édition imprimée

Date de publication : 31 décembre 2015

Pagination : 185-199

ISBN : 978-2-7535-4776-6

ISSN : $1960-1360$

Référence électronique

Thomas Delbey, Alejandro Quevedo et Fanette Laubenheimer, «Amphores vinaires Gauloise 4 de Narbonnaise (France) exportées à Carthagène (Espagne) ? Analyse statistique exploratoire multidimensionnelle de données géochimiques », ArcheoSciences [En ligne], 39 | 2015, mis en ligne le 31 décembre 2017, consulté le 01 février 2022. URL : http://journals.openedition.org/archeosciences/ 4488 ; DOI : https://doi.org/10.4000/archeosciences.4488 


\title{
Amphores vinaires Gauloise 4 de Narbonnaise (France) exportées à Carthagène (Espagne)? Analyse statistique exploratoire multidimensionnelle de données géochimiques
}

\author{
Wine Amphore Gauloise 4 from Narbonnensis (France) Exported \\ to Cartagena (Spain)? Multidimensional Statistical Analysis of Geochemical Data
}

\author{
Thomas Delbey $^{\mathrm{a}}$, Alejandro Quevedo ${ }^{\mathrm{b}}$ et Fanette Laubenheimer ${ }^{\mathrm{c}}$
}

\begin{abstract}
Résumé : La vaste distribution des amphores de type Gauloise 4 dans le monde méditerranéen durant le Haut-Empire témoigne de l'intense activité du commerce vinaire de la Narbonnaise durant cette période. La diffusion de ce type d'amphore dans la Péninsule ibérique, attestée par quelques amphores, demeure encore mal connue. La récente découverte à Carthagène de plusieurs fragments de Gauloise 4 présentant de fortes ressemblances avec les productions de Narbonnaise nous a conduits à réaliser une série d'analyses chimiques afin de nous assurer de leur provenance. Ainsi, huit amphores ont été analysées par fluorescence de rayons-X et comparées à la base de données de la Maison de l'Orient de la Méditerranée, riche de plus de 700 résultats d'analyses d'amphores produites dans le sud de la France. Les données ont ensuite été traitées en utilisant une méthode statistique de partitionnement mixte combinant l'Analyse en composante principale et l'algorithme des Nuées dynamiques (dérivé de la méthode K-means). Les résultats ont ensuite été validés par une analyse factorielle discriminante associée à une validation croisée. Les probabilités d'attributions obtenues nous ont permis de conclure qu'au moins cinq des huit échantillons ont été fabriqués dans les ateliers de Narbonnaise.
\end{abstract}

\begin{abstract}
Strength of wine trade during early Roman Empire is evidenced by the wide geographical distribution of Gauloise 4 amphorae crafted in Narbonnensis area. Extension of that trade to Iberian Peninsula has previously been recorded, but remains very poorly understood. Recent discovery in Cartagena of several pottery sherds closely similar to Gaulish productions led us to investigate their provenance. Eight different amphorae sherds were analyzed using X-ray fuorescence and compared to a solid database of more than 700 amphorae samples produced in south of France. Data was then analyzed by a hybrid clustering method combining Principal component analysis and Dynamic cloud algorithms derived from K-means method. Results were subsequently validated with a Factorial discriminant analysis associated to a cross-validation method. The very high probability of attribution we obtained allowed us to conclude that at least five of the eight samples were produced in workshops located in the Narbonnensis area.
\end{abstract}

Mots clés : Amphores vinaires Gauloise 4, Analyses par fluorescence X, Analyses statistiques, Étude de provenance, Narbonnaise, Carthagène.

Keywords: Gauloise 4 wine Amphorae, X-ray Fluorescence, statistical analysis, clustering analysis, provenance studies, Narbonnensis, Cartagena.

\footnotetext{
a Doctorant, UMR 7041, ArScan, Paris Ouest-Nanterre, équipe GAMA-CNRS.

${ }^{b}$ Chercheur post-doctorant, Aix-Marseille Université, CNRS, UMR 7299, CCJ, LabexMed. La contribution d'A. Quevedo a été réalisée dans le cadre du laboratoire d'excellence LabexMed - Les sciences humaines et sociales au coeur de l'interdisciplinarité pour la Méditerranée portant la référence 10-LABX0090, avec une aide de l'État gérée par l'Agence Nationale de la recherche au titre du projet Investissements d'Avenir A*MIDEX portant la référence no ANR-11-IDEX-0001-02. En même temps la contribution d'A. Quevedo sinscrit dans le cadre du projet de recherche DGICYT (HAR 2011-29330/ HIST): "Carthago Nova: topografía y urbanistica de una urbe mediterránea privilegiada ", financé avec des fonds FEDER.

c UMR 7041, ArScan, Paris-Ouest-Nanterre, équipe GAMA.
} 


\section{INTRODUCTION}

La vitalité du vignoble de Narbonnaise sous le HautEmpire est aujourd'hui bien affirmée par les témoignages des traces de vignoble, des villas viticoles avec leurs chais et des nombreux ateliers d'amphores assurant le conditionnement pour l'exportation lointaine du vin du Midi. L'emballage de prédilection pour le grand commerce était l'amphore Gauloise 4. De telles amphores ont été retrouvées par milliers sur les grands marchés de l'Empire, principalement en Italie, à Ostie et à Rome en particulier, mais aussi vers le limes germanique et l'Angleterre.

Des exportations plus sporadiques ont atteint le bassin méditerranéen oriental dans sa totalité et même les confins de l'Empire comme Palmyre, oasis marchand du désert syrien, ou encore la côte égyptienne de la mer Rouge, voire au-delà vers la corne de l'Afrique et l'Inde du sud (Laubenheimer à paraitre et Laubenheimer, 2015, figure 59).
Mais qu'en est-il des exportations vers le sud-ouest méditerranéen et la Péninsule ibérique? Jusqu’à peu elles étaient mal connues et ce n'est que récemment que quelques exemplaires d'origine gauloise présumée ont attiré l'attention des chercheurs. Dans les îles Baléares, , C. Panella en indiquait déjà la présence à Majorque (Panella 1973, 543), et tout récemment elle est signalée à Ibiza (Laubenheimer à paraitre). Plusieurs exemplaires sont aussi identifiés au Portugal, dans l'ancienne Lusitanie (Filipe 2013). On compte également des importations gauloises à Hispalis (Séville), amphores Gauloise 4 et céramique sigillée de La Graufesenque (García Vargas 2012). Vers le détroit de Gibraltar c'est le cas de Belo où plusieurs amphores Gauloise 4 dont les timbres assurent la provenance de Narbonnaise ont été trouvées (Laubenheimer à paraître). Enfin au-delà, le point extrême des exportations occidentales connues se situe à Lixus, sur la côte atlantique nord de la Maurétanie Tingitane (Maroc) (Aranegui, Hassini 2010) où une anse

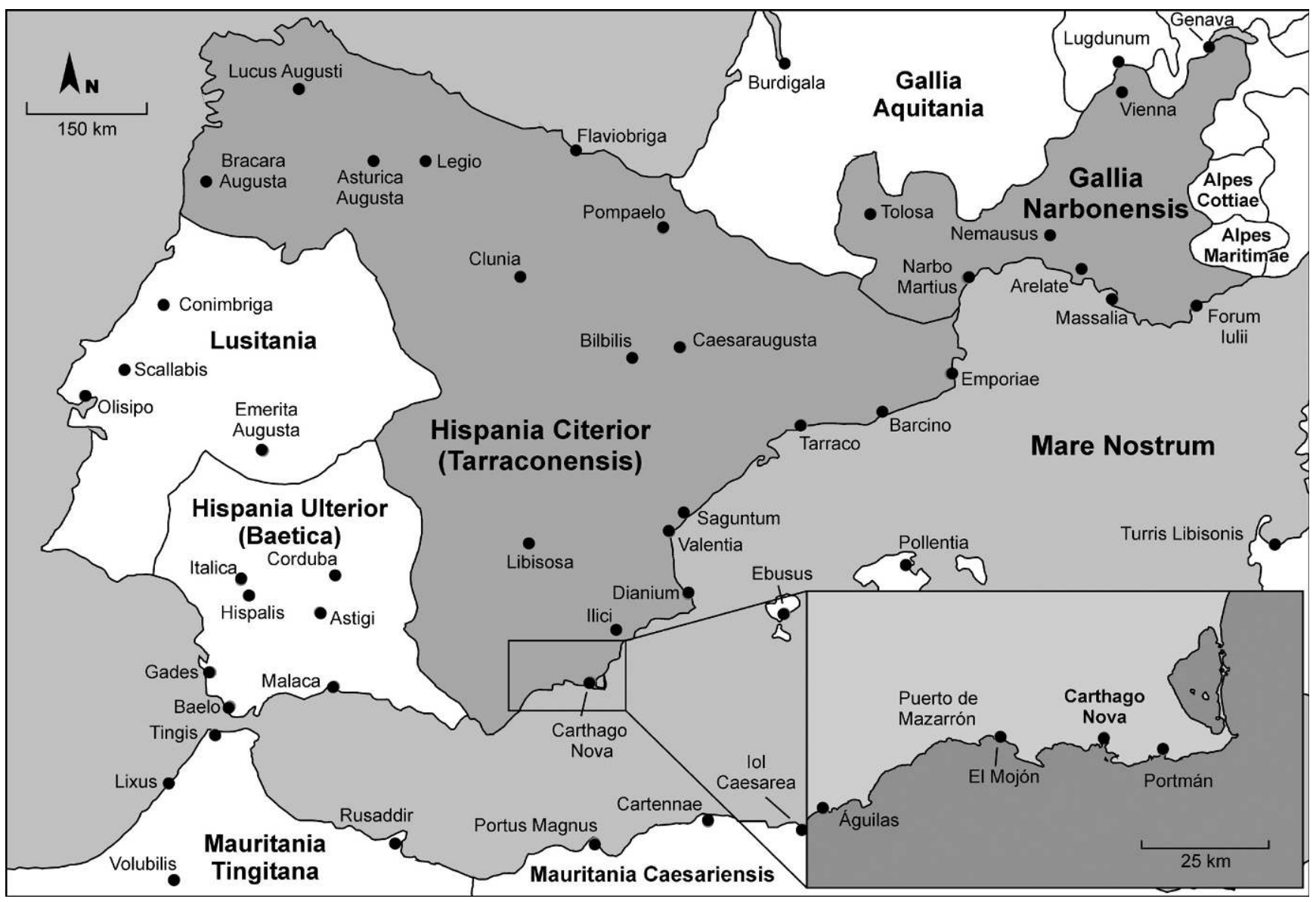

Figure 1 : Carte de situation.

Figure 1: Localization map. 
d'amphores Gauloise 4 timbrée APM ne laisse aucun doute sur son origine, elle provient de la vallée du Rhône.

Les traces en pointillé de ce commerce pourraient bien trouver une nouvelle confirmation sur la côte sud-est de la Péninsule ibérique, à Carthagène et dans ses environs. La question a été posée par l'un de nous, A. Q., qui a observé dans plusieurs fouilles de Carthagène et de la villa Portmán, située à $13 \mathrm{~km}$ de l'ancienne colonie (figure 1), la présence de cols et de fonds dont la facture et la pâte pouvaient correspondre à celle des produits de Narbonnaise plutôt qu'à celle d'imitations locales (Quevedo 2015 s.p.). L'absence d'estampille ou de marque peinte ne pouvait pas confirmer leur origine. En revanche, nous avions à notre disposition deux outils pour vérifier l'origine narbonnaise de ces productions : la base de données d'échantillons photographiques des pâtes des ateliers d'amphores gaulois "Terres d'amphores " (http:// www.mae.u-paris 10.fr/terresdamphores/) et surtout la base de données analytiques des ateliers de Narbonnaise réalisée au Laboratoire de Céramologie de Lyon (Laubenheimer, Schmitt 2009) accessible en ligne (http://amphores.mom. $f r$ ). Cette base de donnée regroupe les résultats des analyses chimiques d'amphores et fournie notamment des informations sur la typologie de l'amphore, le type de pâte, son statut et son attribution.

Dans un premier temps, l'étude et la comparaison macroscopique des pâtes des échantillons de Carthagène avec celles des ateliers de Narbonnaise a permis de proposer l'hypothèse d'une origine gauloise pour ces tessons découverts en Espagne. L'objectif de notre étude est de confirmer cette hypothèse et de tenter d'attribuer ces échantillons à un atelier ou une zone de production spécifique, en comparant leurs données géochimiques à celles des ateliers de référence enregistrées dans la base de données du laboratoire de Céramologie de Lyon. L'approche proposée repose sur l'utilisation d'une méthode de traitement statistique combinant les capacités descriptive des analyses factorielles, la robustesse des méthodes de partitionnement itératives et le pouvoir prédictif de l'analyse discriminante permettant de quantifier la qualité des attributions proposée. Alejandro Quevedo a fourni les échantillons nécessaires et leur documentation. Thomas Delbey s'est chargé d'analyser les résultats sous le contrôle d'Anne Schmitt, directrice du Laboratoire de Céramologie de Lyon.

\section{2. ÉCHANTILLONNAGE}

Huit échantillons d'amphores de type Gauloise 4 (figure 2 et 3 , tableau 1) ont été sélectionnés dans trois fouilles différentes de Carthagène ou de sa proximité immédiate.
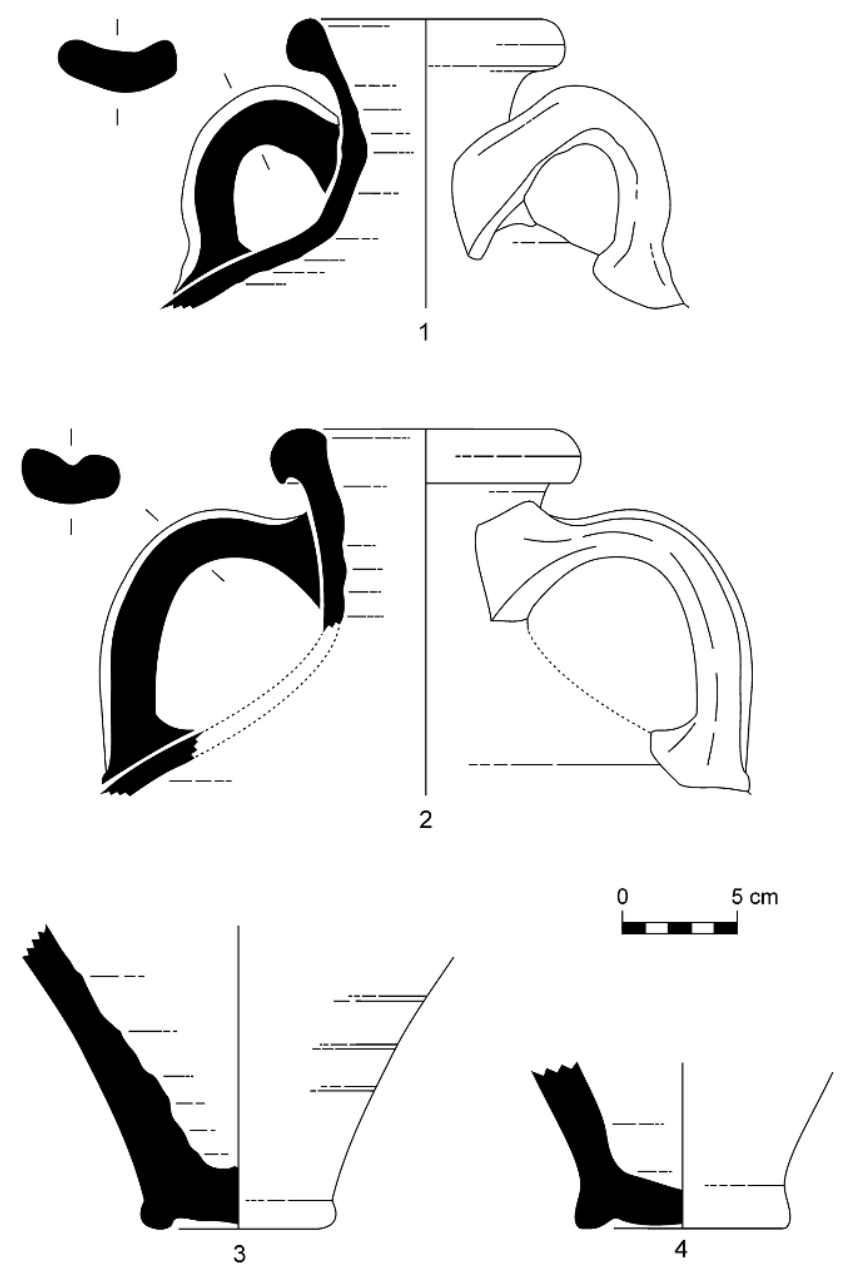

Figure 2 : Amphores Gauloise 4 de la Villa Portmán. Figure 2: Gauloise 4 amphorae from Villa Portman.

Ces échantillons ont été choisis en raison de leur forme (Gauloise 4), rare dans cette région, ainsi que pour la ressemblance macroscopique de leurs pâtes avec les productions des ateliers de Narbonnaise.

Les trois contextes correspondent à une période où la production d'amphores gauloises de Narbonnaise est encore active. Toutefois, on ne voit plus arriver alors dans cette région de l'Espagne la vaisselle gauloise de La Graufesenque (Millau), bien qu'elle perdure dans des contextes de la fin du II ${ }^{\mathrm{e}}$ siècle et du début du III ${ }^{\mathrm{e}}$ siècle. 

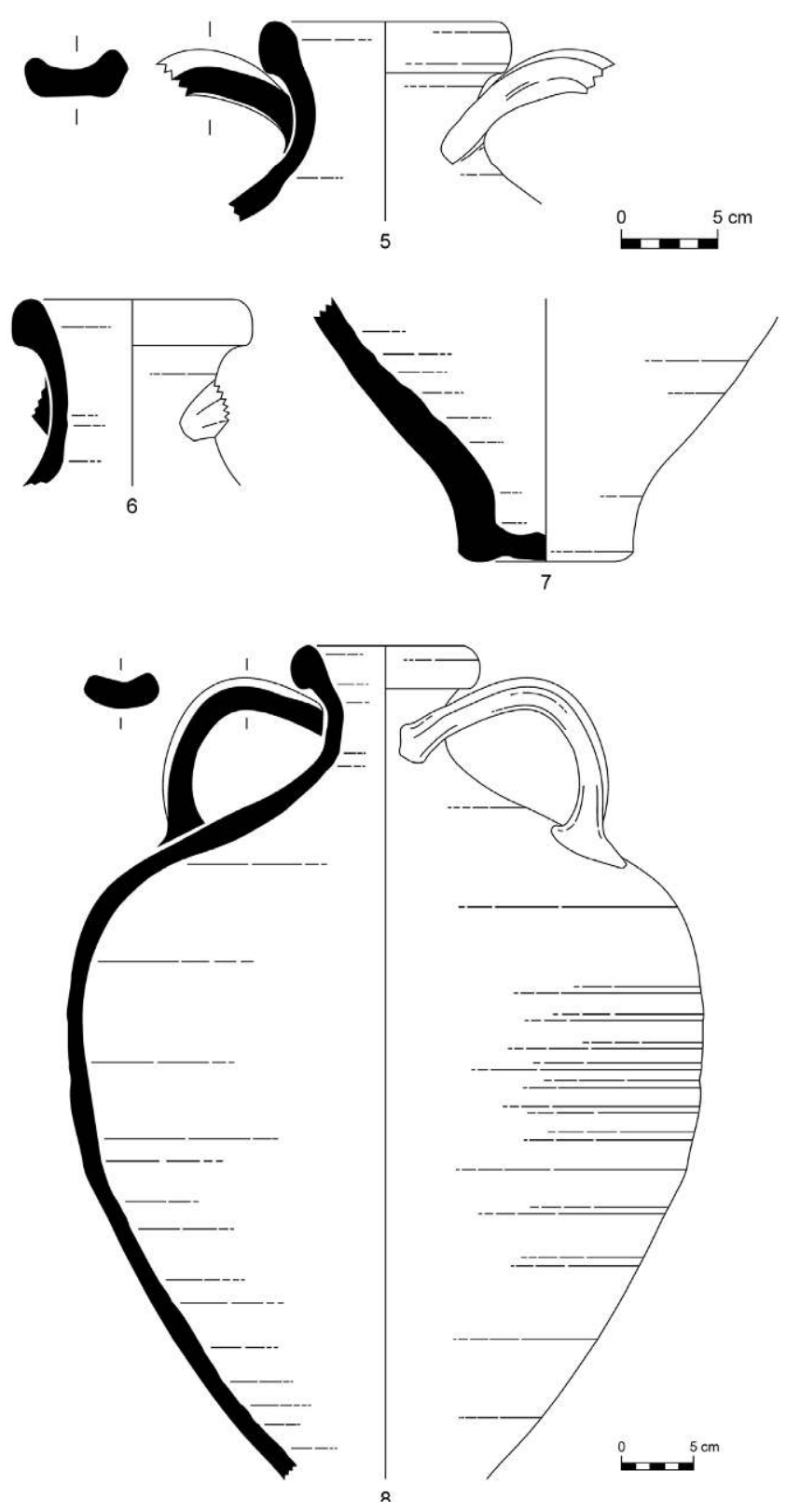

Figure 3 : Amphores Gauloise 4 de la Domus de la Fortuna et de la rue Serreta.

Figure 3: Gauloise 4 Amphorae from Domus of Fortuna and Serreta Street.

\section{Méthode}

\section{Analyse par spectrométrie de fluorescence $\mathrm{X}$ à dispersion de longueur d'onde}

Les échantillons de la base de données analytiques des amphores vinaires de Narbonnaise et les huit échantillons de Carthagène ont été analysés au Laboratoire de Céramologie de Lyon par spectrométrie de fluorescence $\mathrm{X}$ à dispersion de longueur d'onde (WD-XRF) sur un spectromètre séquentiel SR3400 Brucker. Cette méthode d'analyse élémentaire globale fournie la composition chimique résultant de l'analyse de la fraction fine $(<2 \mu \mathrm{m})$ et de la fraction grossière $(>2 \mu \mathrm{m})$ d'un échantillon. Le dosage de 24 éléments chimiques (10 éléments majeurs et 14 éléments traces) est effectué sur une seule perle de verre par échantillon. La préparation des échantillons comprend une étape de décapage de la surface de l'échantillon à la scie diamantée, une étape de grillage à $950^{\circ}$ pour éliminer les traces de $\mathrm{CO}^{2}, \mathrm{~d}^{\prime} \mathrm{H}_{2} \mathrm{O}$ et de matières organiques éventuellement présentes dans les échantillons, une étape de broyage, et une dernière étape de fusion entre l'échantillon broyé et un fondant (tétraborate et métaborate de lithium) afin de créer une " perle de verre " dont la surface est ensuite analysée dans le spectromètre. Les résultats de quantification élémentaire sont ensuite corrigés par des algorithmes pour pallier aux effets de matrice.

\section{Analyse des données}

\section{Tri dans la base de données analytique}

En amont du traitement statistique des données, un premier tri des échantillons de comparaison de la base de données analytique est réalisé. La problématique de la recherche de provenance impose travail de comparaison avec des amphores dont l'attribution d'origine est robuste. Les échantillons sélectionnés proviennent donc tous des sites de productions et non de sites de consommation. Dans la mesure du possible, ces échantillons proviennent d'amphores de type Gauloise 4, comme celle de Carthagène. Cependant, comme certains ateliers sont trop peu représentés par ce type, des échantillons provenant d'amphores de type Gauloise 5 ou d'autres amphores à fond plat sont également sélectionnés. La qualité de la représentation des compositions chimiques par rapport à l'ensemble des échantillons de l'atelier où du groupe d'atelier est prise en compte. En utilisant le calcul des distances de Mahalanobis sur une classification ascendante hiérarchique, Fanette Laubenheimer et Anne Schmitt ont repéré des échantillons provenant de sites de production dont la composition est qualifiée de marginale par rapport au groupe de référence (Laubenheimer, Schmitt, 2009). Ces individus sont écartés de l'étude. La nature " calcaire " des pâtes des amphores de Carthagène, déterminée par l'observation macroscopique et les résultats de l'analyse WD-XRF, nous permet de ne sélectionner que des échantillons dont la pâte est également qualifiée de "calcaire ". Au final, la sélection d'échantillons de référence pour la comparaison après ce tri comprend deux cents cinquante-quatre indi- 


\begin{tabular}{|c|c|c|c|c|c|}
\hline Site & Contexte chrono. & Type & $\mathrm{N}^{\circ}$ inventaire & $\begin{array}{l}\mathrm{N}^{\circ} \text { analyse et } \\
\text { figure }\end{array}$ & Conservation \\
\hline $\begin{array}{l}\text { Villa Portmán } \\
\text { (près de Carthagène) }\end{array}$ & 193-220 apr. J.-C. & $\mathrm{Col}$ & R14 & $\begin{array}{l}\text { AMG } 798 \\
\text { Figure } 2.1\end{array}$ & Musée archéologique de Murcie \\
\hline $\begin{array}{l}\text { Villa Portmán } \\
\text { (près de Carthagène) }\end{array}$ & 193-220 apr. J.-C. & $\mathrm{Col}$ & $0 / 6643$ & $\begin{array}{l}\text { AMG } 799 \\
\text { Figure } 2.2\end{array}$ & Musée archéologique de Murcie \\
\hline $\begin{array}{l}\text { Villa Portmán } \\
\text { (près de Carthagène) }\end{array}$ & 193-220 apr. J.-C. & Fond & R15 & $\begin{array}{l}\text { AMG } 800 \\
\text { Figure } 2.3\end{array}$ & Musée archéologique de Murcie \\
\hline $\begin{array}{l}\text { Villa Portmán } \\
\text { (près de Carthagène) }\end{array}$ & 193-220 apr. J.-C. & Fond & $0 / 6563$ & $\begin{array}{l}\text { AMG } 801 \\
\text { Figure } 2.4\end{array}$ & Musée archéologique de Murcie \\
\hline $\begin{array}{l}\text { Domus de la Fortuna } \\
\text { (Carthagène) }\end{array}$ & 180-210 apr. J.-C. & $\mathrm{Col}$ & H-I-ib'159.1 & $\begin{array}{l}\text { AMG } 802 \\
\text { Figure } 3.5\end{array}$ & $\begin{array}{c}\text { Musée archéologique municipal } \\
\text { de Carthagène }\end{array}$ \\
\hline $\begin{array}{l}\text { Domus de la Fortuna } \\
\text { (Carthagène) }\end{array}$ & 180-210 apr. J.-C. & $\mathrm{Col}$ & CD-1110-127.2 & $\begin{array}{l}\text { AMG } 803 \\
\text { Figure } 3.6\end{array}$ & $\begin{array}{c}\text { Musée archéologique municipal } \\
\text { de Carthagène }\end{array}$ \\
\hline $\begin{array}{l}\text { Domus de la Fortuna } \\
\text { (Carthagène) }\end{array}$ & 180-210 apr. J.-C. & Fond & A.RE 159.2 & $\begin{array}{l}\text { AMG } 804 \\
\text { Figure } 3.7\end{array}$ & $\begin{array}{c}\text { Musée archéologique municipal } \\
\text { de Carthagène }\end{array}$ \\
\hline $\begin{array}{l}\text { Rue Serreta 8-10-12 } \\
\text { (Carthagène) }\end{array}$ & Fin $\mathrm{II}^{\mathrm{e}} s .-$ Début $\mathrm{III}^{\mathrm{e}} s$. & $\begin{array}{l}\text { Forme } \\
\text { Entière }\end{array}$ & Sans $n^{\circ}$ & $\begin{array}{l}\text { AMG } 805 \\
\text { Figure } 3.8\end{array}$ & $\begin{array}{c}\text { Musée archéologique municipal } \\
\text { de Carthagène }\end{array}$ \\
\hline
\end{tabular}

Tableau 1 : Échantillonnage des amphores Gauloise 4 de Carthagène

Table 1: Sampling of the Gaulish 4 amphorae from Carthagena.

vidus provenant de vingt-six ateliers ou groupes d'ateliers (les différents ateliers d'une même commune actuelle sont regroupés) localisés en Narbonnaise au haut Empire.

Les résultats des analyses élémentaires sont également triés afin d'écarter les éléments chimiques dont l'origine peut être dû à la pollution des sols. Au final, seize éléments chimiques sont conservés, huit éléments majeurs $\left(\mathrm{CaO}, \mathrm{Fe} 2 \mathrm{O} 3, \mathrm{TiO}_{2}\right.$, $\mathrm{K}_{2} \mathrm{O}, \mathrm{SiO}_{2}, \mathrm{Al}_{2} \mathrm{O}_{3}, \mathrm{MgO}$ et $\left.\mathrm{MnO}\right)$ et huit éléments traces $(\mathrm{Zr}$, $\mathrm{Sr}, \mathrm{Rb}, \mathrm{Zn}, \mathrm{Cr}, \mathrm{Ni}$, V et $\mathrm{Ce}$ ).

\section{Protocole d'analyse statistique}

1) Le protocole d'analyse statistique utilisé pour traiter les données a pour but de créer un modèle de classification des amphores de Narbonnaise à partir des critères de composition chimique élémentaire et d'évaluer la similarité entre cette classification et les attributions des échantillons par atelier ou par zone de production. Ensuite, les huit échantillons de Carthagène sont intégrés au modèle pour évaluer leurs correspondances avec les classes construites et proposer une attribution d'atelier ou de zone de production. Le protocole comprend trois étapes nécessaires à la description et la classification des données.

La réalisation d'une analyse en composantes principales (ACP de Person) est faite sur une matrice de données centrée réduite des dosages des seize éléments chimiques obtenus sur l'ensemble des 261 échantillons, soit sur 253 échantillons de comparaison illustrant les ateliers ou zones de production et huit échantillons provenant de Carthagène. Ces derniers sont intégrés dans l'analyse en tant qu' « individus supplémentaires " afin qu'ils soient projetés dans l'espace factoriel mais sans influencer sur sa construction. L'ACP permet, en regroupant sur la base de leurs corrélations les variables initiales en un nombre réduit de variables synthétiques, d'obtenir une représentation des données dans un espace multi-dimensionnel. L'analyse des résultats passe ensuite par le choix des axes factoriels qu'il est pertinent de conserver pour l'interprétation des données. Plusieurs critères de sélection des axes existent pour faciliter leur choix, comme le critère de Kaiser (Kaiser, 1960) ou celui de Cattell (Cattell, 1966). Bien que ces indices soient pris en considération, c'est, au final, la possibilité d'interpréter les axes qui détermine leur sélection. Les plans factoriels les plus importants peuvent ensuite être représentés graphiquement. Une analyse en classification ascendante hiérarchique (CAH) sur les coordonnées des variables les regroupe en pôles autour desquels les individus sont distribués et facilite l'interprétation des axes factoriels. La proximité des variables entre elles traduit une corrélation positive qui dépend de la nature des faciès géologiques exploités pour les matières premières et leurs préparations par les potiers.

D'autres méthodes, comme l'analyse Log-Ratio (LRA) (Aitchison, 1986) sont utilisées dans les analyses de compositions élémentaires et fournissent des résultats plus adaptés pour une interprétation détaillée des compositions chimiques. L'objectif de cette étude portant principalement sur la classification d'individus, l'analyse des structures de covariances de l'A.CP. propose une approche plus simple et plus directe, au risque d'une certaine perte d'information. La 
comparaison de ces deux méthodes sur du mobilier archéologique (Baxter et al., 2005) met en évidence la similarité de la classification obtenue.

2) Le choix du nombre de classes et la classification des individus par une méthode de classification mixte (Lebart $e t$ al., 2006), adaptation de l'Hybrid Clustering (par ex. Wong, 1982), d'après leurs coordonnées en A.C.P. sur les axes factoriels retenus.

Cette méthode combine la classification ascendante hiérarchique $(\mathrm{CAH})$ et l'algorithme de partitionnement itératif des nuées dynamiques (ND) (Diday, 1971). L'utilisation d'une méthode de partitionnement itératif propose une classification plus robuste que celles des méthodes d'agrégation hiérarchiques seules (Tan et al., 2006; Boutsidis et al., 2009).

Cette approche mixte passe par une première classification des nuées dynamiques sur les 253 échantillons de référence, contrainte à un nombre de partition égale au nombre d'ateliers de références analysés (trente ateliers ou groupes d'ateliers). Ensuite, une analyse CAH est appliquée sur les barycentres des classes déterminées par l'analyse des ND, afin de choisir le nombre optimal $K$ de classes à créer. Pour déterminer au mieux ce nombre, la CAH est répétée trente fois avec, à chaque itération $i$, un découpage en $i+1$ classe. L'évolution de la variance de l'inertie intra-classe et inter-classe en fonction du nombre des $K$ classes permet de calculer plusieurs indices utiles pour déterminer le nombre de classes :

- Indice du $\mathrm{R}^{2}$ : Ce critère détermine la proportion d'inertie expliquée par les classes. Sur la courbe du $\mathrm{R}^{2}$, le point de rupture de la courbe formant un " coude " à $k$ classes représente le nombre de classe déterminé par ce critère. Il se calcule de la façon suivante :

$$
R^{2}=1-\frac{\text { Iintra }(P k)}{\text { Itotale }}=\frac{\text { Iinter }(P k)}{\text { Itotale! }}
$$

- Indice du SPRSQ (Semi partial R Square) : Ce critère détermine la perte d'inertie inter-classe provoquée par le regroupement de deux classes. Le nombre de classes à choisir se trouve au point de la courbe qui marque la plus forte réduction. Ce critère se calcule de la façon suivante :

$$
S P R S Q=\frac{\operatorname{Iintra}(P k)-\operatorname{Iinter}(P k-1)}{\text { Itotale }}
$$

- Indice du Pseudo F : Ce critère détermine le degré de séparation entre les classes. Sur la courbe, un pic sensible entre deux $k$ classes indique un point possible pour déterminer le nombre de classes. Ce critère se mesure de la façon suivante :

$$
\text { Pseudo } F=\frac{\text { Iinter }(P k) /(K-1))}{\operatorname{Iintra}(P k) /(n-K)}
$$

Outre ces critères statistiques, des critères archéologiques sont utilisés pour affiner ces résultats et faire un choix dans le nombre de classes proposé par les critères statistiques. Des valeurs nominales sont attribuées aux échantillons de référence en fonction de leur atelier ou de leur zone de production (d'après le découpage en zone dans Laubenheimer, Schmitt, 2009). Ces valeurs sont ensuite comparées aux résultats des classifications en fonction du nombre de classes potentiel déterminées par les critères statistiques grâce à la construction d'un tableau de contingence qui permet de mesurer la dépendance entre les attributions statistiques et archéologiques via les scores du test $\mathrm{khi}^{2}$ et l'inertie des classes (calculée sur le modèle de l'inertie de l'analyse factorielle des correspondances).

Une fois le nombre de classes fixé, une seconde classification des nuées dynamiques est ensuite réalisée sur les coordonnées de tous les échantillons (y compris ceux de Carthagène), en définissant comme valeurs initiales des centres des classes les barycentres des centroïdes des $K$ classes choisies préalablement. Les échantillons classifiés sont ensuite projetés sur le plan factoriel de l'ACP pour leur description, et un test du khi ${ }^{2}$ et un test exact de Fisher permettent de déterminer si une ou plusieurs classes sont représentatives d'ateliers proches ou d'une zone de production particulière. A partir de cette étape, il est possible de fournir une proposition d'attribution pour les échantillons de Carthagène.

3) L'estimation du taux d'erreur des attributions de la classification mixte et l'évaluation de la qualité du modèle de partitionnement via une analyse factorielle discriminante (AFD) : cette méthode fournit également des prédictions d'attributions des individus a posteriori permettant de corriger le modèle des classes proposées a priori.

Toutefois, puisqu'il a été démontré que l'AFD surévalue systématiquement la qualité du modèle (Kovarovic et al., 2011), il est indispensable de corriger cette tendance par une validation croisée par LOOCV (Leave one-out cross validation). Cet outil évalue la validité du modèle en croisant les résultats obtenus après exclusion de chaque individu un par un et permet d'obtenir une évaluation jugée plus réaliste (Bernau et al. 2014).

\section{RÉSUltats}

\section{Choix et interprétations des facteurs de l'ACP}

L'histogramme d'éboulement des valeurs propres des axes de l'A.C.P. (figure 4) montre une décroissance rapide des valeurs propres des quatre premiers axes. Leurs valeurs 


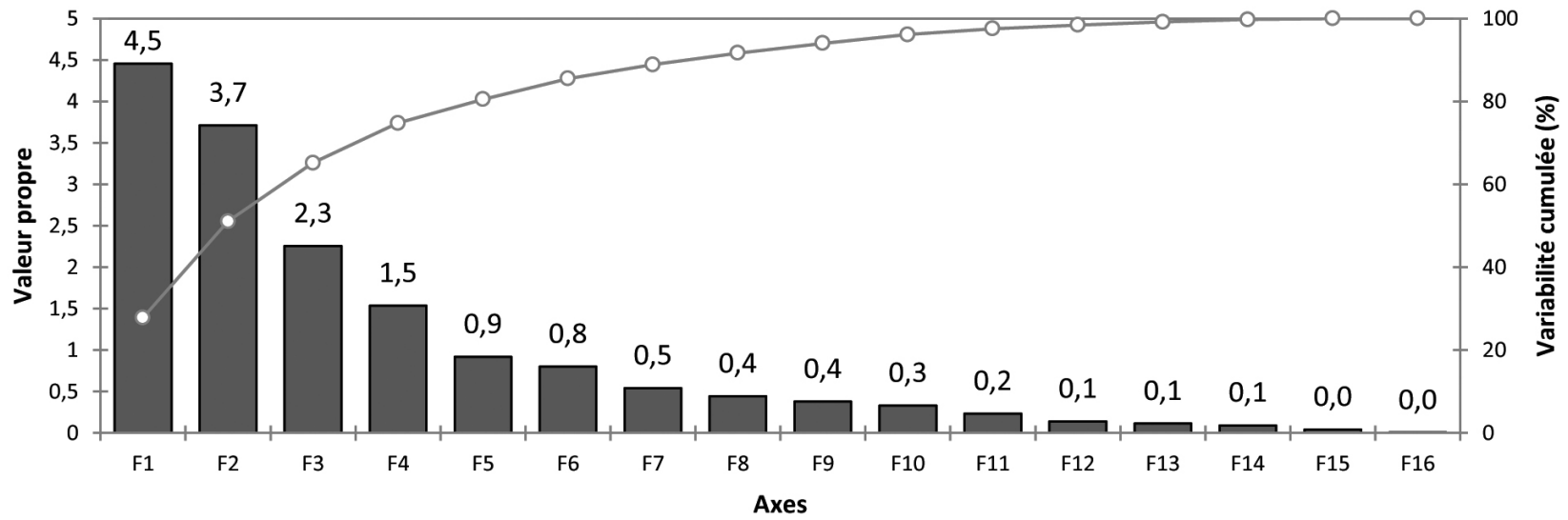

Figure 4 : Histogramme d'éboulement des valeurs propres des axes de l'A.CP. Figure 4: Scree plot of the eigenvalue of P.C.A.

propres sont supérieures à 1 (critère de Kaiser) et un décrochement suivi d'une décroissance régulière sont également visibles à partir de l'axe 4 (critère de Cattell).

L'observation du tableau de corrélation des variables aux axes factoriels permet de déterminer quels axes sont interprétables. Seuls les trois premiers axes ont plus d'une variable présentant une valeur de contribution significative $(>0,5)$. De plus, toutes les variables, à l'exception de $\mathrm{Zn}$ ont un rapport de corrélation significatif avec l'un des trois premiers axes. L'interprétation des résultats de l'ACP se base donc sur ces trois premiers axes et permet d'établir les relations entre les éléments chimiques qui constituent les pôles autour desquels les échantillons se dispersent. En l'absence d'analyse minéralogique ou granulométrique, il est difficile d'interpréter plus précisément l'origine des éléments dosés par fluorescence $\mathrm{X}$. D’après les résultats de la CAH sur les coordonnées des variables, quatre pôles se distinguent nettement (figure 5) :

- Le premier pôle est riches en fer ( $\mathrm{Fe} 2 \mathrm{O} 3)$, en manganèse $(\mathrm{MnO})$ et en éléments traces sidérophiles ( $\mathrm{V}, \mathrm{Ni}$ et $\mathrm{Cr}$ ). Les rayons ioniques du fer et du manganèse étant très proche, la substitution entre les deux est très fréquente dans les silicates (Papin, 2001). La corrélation entre ces deux éléments est donc souvent constatée dans les sédiments.

- Le second pôle est riche en calcium $(\mathrm{CaO})$ et en strontium (Sr). La forte corrélation entre ces deux éléments est généralement caractéristique des roches d'origine marine.

- Le troisième pôle caractérise les échantillons riches en aluminium $\left(\mathrm{Al}_{2} \mathrm{O}_{3}\right)$, potassium $\left(\mathrm{K}_{2} 0\right)$, titane $\left(\mathrm{TiO}_{2}\right)$ et en rubidium $(\mathrm{Rb})$. La présence de feldspaths résistant à l'altération peut être à l'origine de la corrélation entre l'aluminium et le potassium. Le rubidium se substitue souvent au potassium dans les feldspaths et dans certains phyllosilicates (Herut, Sandler, 2006)
- Le quatrième pôle caractérise les échantillons riches en silicium $(\mathrm{SiO} 2)$, zirconium $(\mathrm{Zr})$ et cérium $(\mathrm{Ce})$. La forte corrélation entre $\mathrm{SiO}_{2}$ et $\mathrm{Zr}$ peut être caractéristique de pâtes avec un dégraissant de sable quartzeux très abondant.

\section{Classification des échantillons et descriptions des classes}

\section{Choix du nombre de classes}

La courbe du critère SPRSQ (figure 6a) montre plusieurs décroissances significatives : la plus importante se trouve entre $\mathrm{k}=4$ et $\mathrm{k}=5$, et deux plus faibles entre $\mathrm{k}=10$ et $\mathrm{k}=11$ et entre $\mathrm{k}=17$ et $\mathrm{k}=18$. Pour le critère Pseudo $\mathrm{F}$, la courbe (figure 6b) montre plusieurs pics : un pic principal à $\mathrm{k}=5$ classes et un deuxième pic à $\mathrm{k}=10$ classes. Sur les courbes des critères de variance inter-classe et du $\mathrm{R}^{2}$ (figure $6 \mathrm{~b}$ ), un léger coude se forme au niveau de $\mathrm{k}=5$ classes pour les deux courbes.

L'analyse d'un tableau de contingence confrontant le découpage des N.D. et le découpage archéologique montre des relations de dépendance forte à $\mathrm{k}=5$ classes, aussi bien pour le découpage en ateliers qu'en zones de productions (tableau 2).

Les relations de dépendance les plus fortes entre le découpage en $k$ classes et les variables archéologiques sont entre les classes de $\mathrm{k}=5$ classes et les attributions par ateliers.

En accord avec les conclusions des analyses statistiques réalisées par A. Schmitt (Laubenheimer et Schmitt, 2009), le nombre de classes déterminé par les critères statistiques ne correspond ni au nombre d'ateliers de références analysés, ni aux zones de productions. La cause en est la grande diversité des matériaux utilisés pour la fabrication des amphores 


\begin{tabular}{|c|c|c|c|}
\hline Variables & F1 & F2 & F3 \\
\hline $\mathrm{V}$ & 0,695 & 0,002 & 0,038 \\
\hline $\mathrm{Ni}$ & 0,714 & 0,098 & 0,136 \\
\hline $\mathrm{Cr}$ & 0,772 & 0,338 & $-0,109$ \\
\hline $\mathrm{MnO}$ & 0,703 & 0,282 & $-0,196$ \\
\hline $\mathrm{Fe} 203$ & 0,824 & $-0,160$ & $-0,375$ \\
\hline $\mathrm{Zn}$ & 0,344 & 0,336 & $-0,374$ \\
\hline $\mathrm{MgO}$ & 0,174 & 0,551 & $-0,570$ \\
\hline $\mathrm{Sr}$ & 0,060 & 0,243 & 0,750 \\
\hline $\mathrm{CaO}$ & $-0,222$ & 0,734 & 0,537 \\
\hline $\mathrm{Al} 203$ & 0,680 & $-0,627$ & 0,098 \\
\hline $\mathrm{TiO} 2$ & 0,424 & $-0,591$ & 0,018 \\
\hline $\mathrm{Rb}$ & 0,321 & $-0,553$ & 0,510 \\
\hline $\mathrm{K} 20$ & 0,450 & $-0,629$ & 0,361 \\
\hline $\mathrm{Ce}$ & $-0,151$ & $-0,610$ & $-0,237$ \\
\hline $\mathrm{SiO} 2$ & $-0,448$ & $-0,528$ & $-0,517$ \\
\hline $\mathrm{Zr}$ & $-0,569$ & $-0,611$ & $-0,073$ \\
\hline
\end{tabular}

Figure 5 : Coordonnées des variables sur les axes factoriels et dendrogramme de la $\mathrm{CAH}$.

Figure 5: Coordinates of the 16 variables on factorial axis and H.A.C. dendrogram.

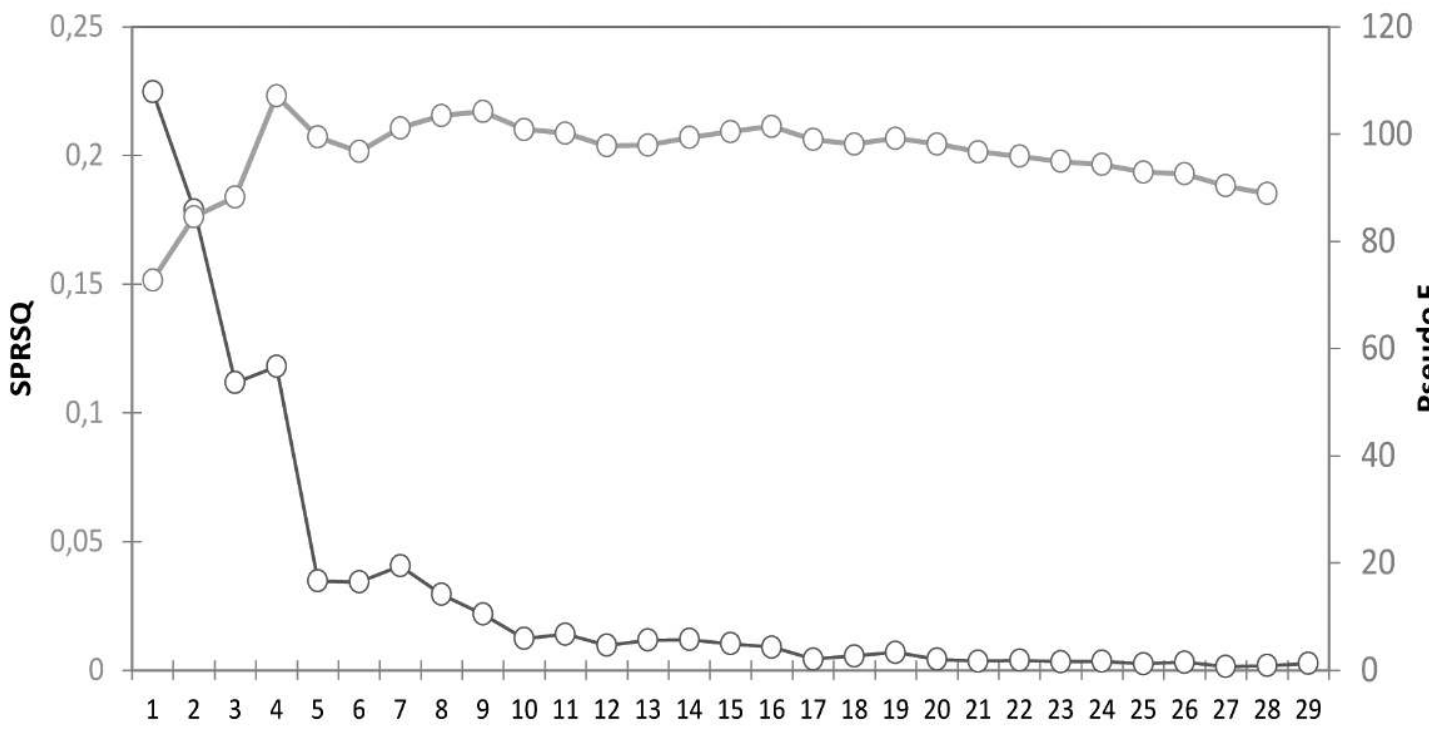

Figure 6a :

Représentation des critères SPRSQ et Pseudo $\mathrm{F}$ en fonction de $\mathrm{K}$ classes. Figure 6a: Figuring of the SPRSQ and Pseudo F criteria depending on $K$ clusters.

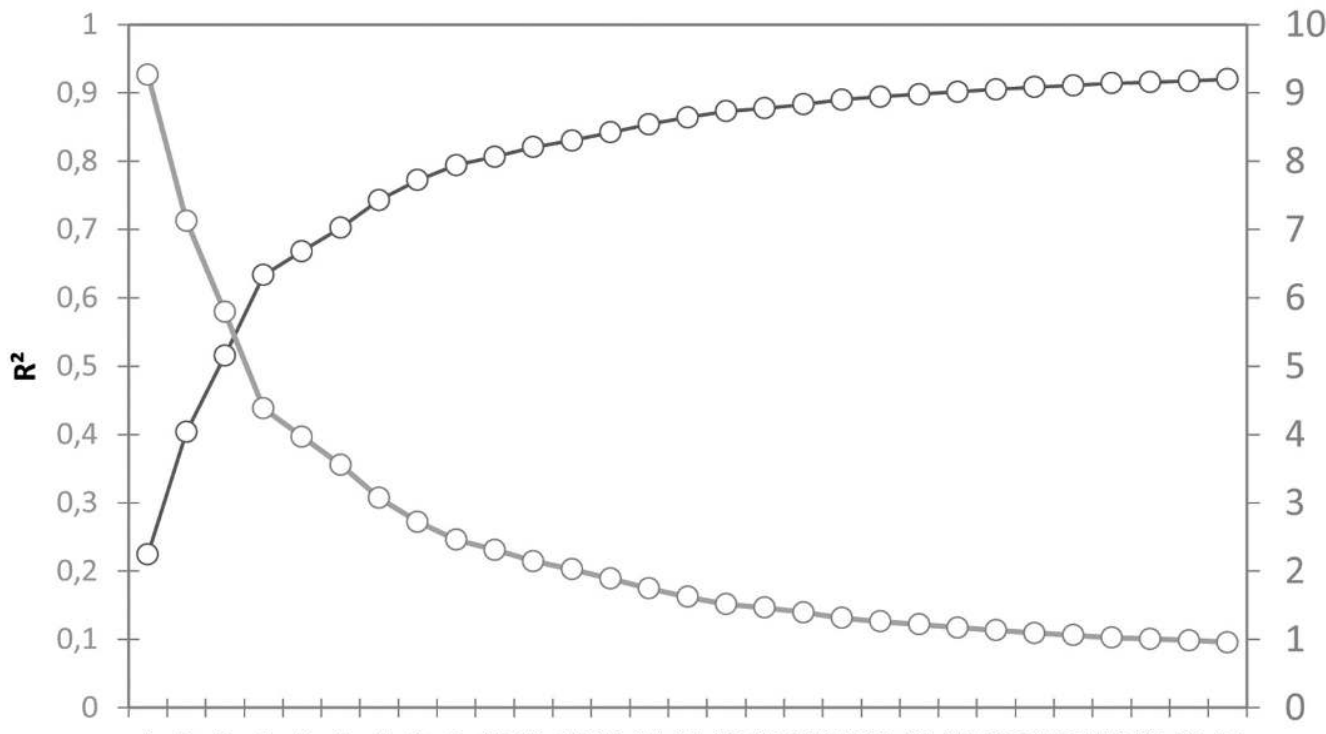

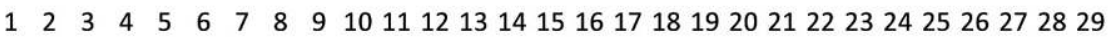

Nombre de classe : $k$
Figure 6b :

Représentation des critères du $\mathrm{R}^{2}$ et de l'inertie inter-classe en fonction de $\mathrm{K}$ classes. Figure 6b: Figuring of the $R^{2}$ and inter-group variation depending on Kclusters. 


\begin{tabular}{|l|c|c|c|c|}
\hline & \multicolumn{2}{|c|}{ Ateliers } & \multicolumn{2}{c|}{ Zones } \\
\hline & $\mathrm{Khi}^{2}$ & Inertie & $\mathrm{Khi}^{2}$ & Inertie \\
\hline $\mathrm{K}=2$ classes & 188,992 & 0,747 & 97,660 & 0,386 \\
\hline $\mathrm{K}=5$ classes & 626,283 & 2,475 & 310,802 & 1,228 \\
\hline $\mathrm{K}=10$ classes & 516,901 & 2,043 & 222,103 & 0,878 \\
\hline $\mathrm{K}=11$ classes & 554,603 & 2,192 & 249,046 & 0,984 \\
\hline
\end{tabular}

Tableau 2 : Relations de dépendance entre le découpage des N.D. et la classification archéologique

Table 2: Dependency ratio between dynamic cloud clustering and archaeological classification.

au sein d'une même région de production et parfois même au sein d'un même atelier (argiles paléogènes et néogènes, sédiments alluviaux ou issus de colluvionnements). Le découpage à $\mathrm{k}=5$ est celui qui revient le plus souvent dans le tableau de synthèse des résultats pour le choix du nombre de classes (tableau 3), et a donc été retenu pour la classification.

\begin{tabular}{|c|c|}
\hline Critères statistiques & $\begin{array}{c}\text { Choix du nombre } \\
\text { de classes }\end{array}$ \\
\hline $\mathrm{R}^{2}$ & 5 classes \\
\hline Inertie Intra & 5 classes \\
\hline SPRSQ & 2,5 ou 11 classes \\
\hline Pseudo $\mathrm{F}$ & 5 ou 10 classes \\
\hline Critères statistiques - archéologiques & \\
\hline $\mathrm{Khi}^{2}$ _ Dépendance $\mathrm{k} /$ ateliers de productions & 5 classes \\
\hline Inertie - Dépendance $\mathrm{k} /$ zones de productions & 5 classes \\
\hline
\end{tabular}

Tableau 3 : Synthèse des résultats pour le choix du nombre de classes

Table 3: Synthesis of the results for choosing the number of groups.

\section{Homogénéité et description des classes}

L'homogénéité des cinq classes découpées peut être qualifiée selon deux critères. Le critère statistique de la variance intra-classe (figure 7) détermine l'homogénéité des signaux géochimiques entre les échantillons d'une même classe. L'homogénéité géographique et archéologique de la provenance des échantillons est le second critère à prendre en compte. Celui-ci est calculé d'après les tests de dépendance du $\mathrm{khi}^{2}$ entre la classification de l'analyse N.D. en $\mathrm{k}=5$ classes et le découpage en ateliers de productions. L'utilisation du test exact de Fisher permet de sélectionner pour chaque classe les ateliers ayant une dépendance significative. Bien qu'une classe puisse avoir une très faible variance intra-classe, si les échantillons qui la composent proviennent de sites éloignés sans relations particulières, elle n'est pas interprétable. Il est alors possible d'effectuer une nouvelle classification à l'intérieur de la classe afin de rechercher un sous-partitionnement qui reflète une logique archéologique. Si cette dernière étape ne fournit pas de résultats satisfaisants, cela signifie que les analyses géochimiques ne sont pas suffisantes pour permettre un classement de ces échantillons.

La représentation des points-individus dans l'espace factoriel des axes 1 et 2 (figure 7) montre une bonne homogénéité des classes et une dispersion qui permet de les différencier et de les décrire en fonction de leurs proximités avec les variables (signaux géochimiques). Les ellipses tracées pour chaque groupe correspondent à un intervalle de confiance de $95 \%$ pour une loi normale bivariée de mêmes moyennes et de même matrice que les variables représentées. Les échantillons de Carthagène se dispersent dans quatre des cinq classes et restent tous à l'intérieur des ellipses de confiances. Seules ces quatre classes sont analysées plus en détails.

\section{- Classe 1}

Sur les graphiques des axes factoriels (figure 5), ces échantillons se démarquent par des valeurs fortes en fer et en manganèse ainsi qu’en éléments traces sidérophiles : le nickel, le chrome et le vanadium. Le nuage de point de ces échantillons est assez ramassé et reflète une variance intra-classe modérée.

Le test de Fisher montre une dépendance positive entre cette classe et la zone de production de la vallée du Gardon (ateliers de Meynes, Montfrin et Vers-Pont-du Gard) et celle de la vallée du Libron (atelier de Laurens). La présence d'échantillons des autres zones n'est pas significative. Trois échantillons de Carthagène (AMG 798, AMG 800 et AMG 803) appartiennent à cette classe.

Une seconde classification par analyse des N.D., suivant la même procédure que le partitionnement principal, est réalisée à l'intérieur de cette classe. Elle propose un partitionnement en cinq sous-classes. La quatrième sous-classe regroupe les trois échantillons de Carthagène de la classe 1 (AMG 798, AMG 800 et AMG 803) ainsi que tous les échantillons des ateliers de Meynes et de Montfrin, et des échantillons moins contribuant : deux de l'atelier de Beaucaire et trois de l'atelier de Saint-Gilles. Ceux-ci se situent dans un rayon de $30 \mathrm{~km}$ au sud de Montfrin et Meynes, proche du Rhône ou de l'un de ses défluents, Le Petit Rhône. Deux autres échantillons, de l'atelier d'Oraison (vallée de la Durance) sont également présents dans cette sous-classe. D'après les résultats des tests de Fisher et du $\mathrm{Khi}^{2}$, les ateliers de Meynes et de Montfrin sont les plus significatifs dans cette sous-classe. La présence de deux ateliers proches de la vallée du Gardon (Saint-Gilles et Beaucaire) atteste de la bonne représentation de cette zone par cette sous-classe et confirme son lien avec les trois échantillons de Carthagène mentionnés. 


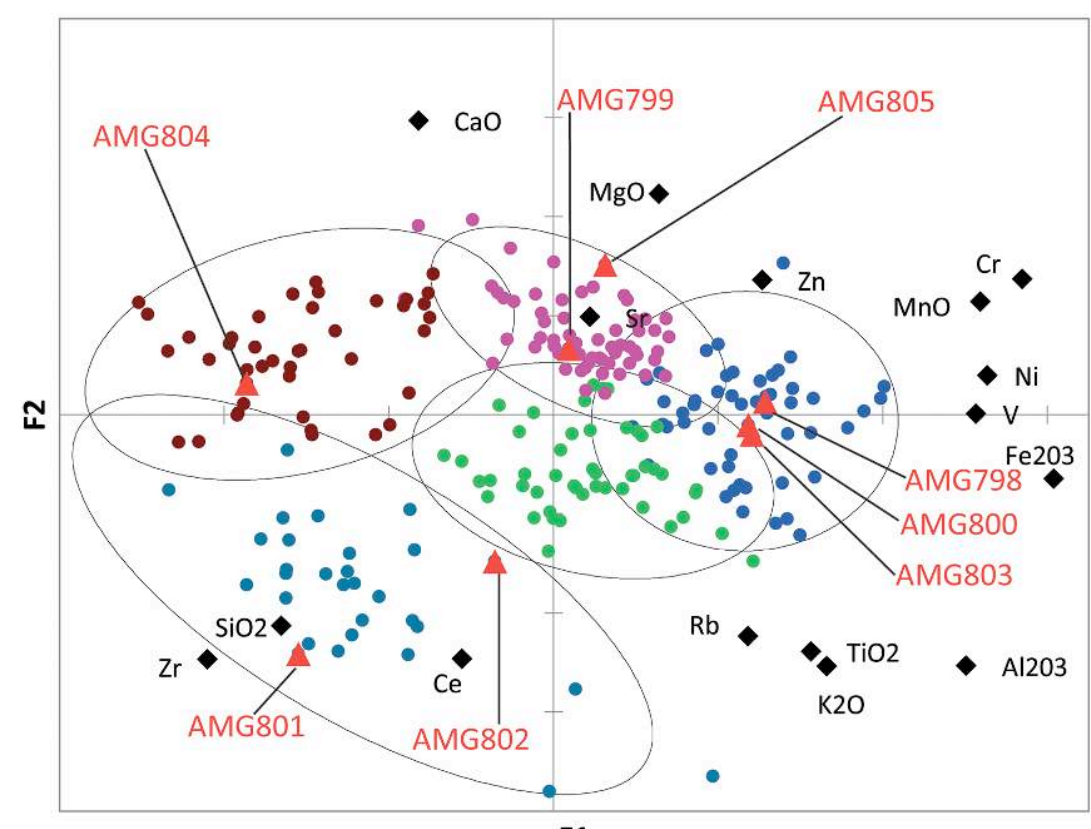

F1

- Classe $1 \bullet$ Classe $2 \bullet$ Classe $3 \bullet$ Classe $4 \bullet$ Classe 5 • Echantillons Carthagène
Figure 7 : (Voir planche couleur XIX) Plan factoriel des axes 1 et 2 avec le découpage en place de l'analyse des nuées dynamiques. Figure 7: (See colour plate XIX) Factorial analysis with dynamic cloud clustering.

\begin{tabular}{|l|c|c|c|c|c|c|c|}
\hline Classe 1 & Vallée de l'Aude & Vallée du Libron & Vallée de l'Hérault & Vallée du Gardon & Vallée du Rhône & $\begin{array}{c}\text { Vallée de la } \\
\text { Durance }\end{array}$ & $\begin{array}{c}\text { PACA } \\
\text { Khi }^{2}\end{array}$ \\
\hline Test de Fischer & $>$ & 3,386 & 7,687 & 30,208 & 0,002 & 0,029 & 4,623 \\
\hline$\%$ inertie & 4,78 & 7,32 & 15,79 & 60,47 & 0,00 & 0,06 & $>$ \\
\hline Effectifs & 8 & 13 & 7 & 16 & 5 & 6,25 \\
\hline
\end{tabular}

Tableau 4 : Résultats pour le test de dépendance de la classe1 à la variable de classification par zone de production Table 4: Dependency ratio between the group 1 and the classification by area of production.

\section{- Classe 3}

Les échantillons de cette classe sont caractérisés par des valeurs très importantes en calcium, strontium et magnésium. Le nuage de point est ramassé et traduit une variance intra-classe faible et une bonne homogénéité des signaux géochimiques.

Le test de Fisher montre une dépendance positive et significative des zones de production de la vallée de de l'Aude, du Rhône et de la Durance. Deux échantillons de Carthagène (AMG 799 et AMG805) sont présents dans cette classe.

La seconde classification de partitionnement de cette classe ne permet pas de dégager de sous-classe cohérente avec le découpage en zones géographiques. Les deux amphores de Carthagène ne peuvent donc pas être reliés à une zone de production spécifique.

\section{Classe 4}

Cette classe est caractérisée par des valeurs faibles en fer, manganèse et aluminium ainsi que pour les éléments traces sidérophiles. La proportion de silice est relativement importante dans tous les échantillons, mais la quantité de carbonate de calcium est très variable au sein de cette classe. Le nuage de points est très éclaté et reflète la forte variance intra-classe. Le test de Fisher montre une dépendance positive entre cette classe et les zones de productions de la vallée de l'Orb (atelier de Vendres) et de la vallée de l'Hérault (atelier de Saint-Pargoire). Un échantillon de Carthagène (AMG 804) est présent dans cette classe. Cette classe apparait comme très représentative de l'atelier de Saint-Pargoire puisque les trente échantillons de la vallée de l'Hérault proviennent de cet atelier. 


\begin{tabular}{|l|c|c|c|c|c|}
\hline $\begin{array}{l}\text { Classe 1 } \\
\text { Sous-classe 4 }\end{array}$ & Oraison & Meynes & Montfrin & Saint-Gilles & Beaucaire \\
\hline Khi $^{2}$ & 0,822 & 7,04 & 5,87 & 3,52 & 0,13 \\
\hline$\%$ inertie & 2,24 & 19,17 & 15,98 & 9,59 & 6,39 \\
\hline Test de Fischer & $>$ & $>$ & $>$ & 3 & $>$ \\
\hline Effectifs & 2 & 6 & 5 & 3 \\
\hline
\end{tabular}

Tableau 5 : Résultats des tests de dépendance de la sous-classe 4 de la classe 1 à la variable de classification par atelier de production. Table 5: Dependency ratio between the sub-group 4 of group 1 and the classification by area of production.

\begin{tabular}{|l|c|c|c|c|c|c|}
\hline Classe 3 & Vallée de l'Aude & Vallée de l'Hérault & Vallée du Gardon & Vallée du Rhône & Vallée de la Durance & PACA \\
\hline $\mathrm{Khi}^{2}$ & 4,384 & 0,020 & 0,772 & 20,208 & 5,261 & 5,800 \\
\hline$\%$ inertie & 9,46 & 0,04 & 1,67 & 43,59 & 11,35 & 12,51 \\
\hline Test de Fischer & $>$ & $<$ & $<$ & $>$ & $<$ \\
\hline Effectifs & 10 & 21 & 3 & 16 & 12 & 4 \\
\hline
\end{tabular}

Tableau 6 : Résultats pour le test de dépendance de la classe 3 à la variable de classification par zone de production. Table 6: Dependency ratio between the group 3 and the classification by area of production.

\begin{tabular}{|l|c|c|c|c|}
\hline Classe 4 & Vallée de l'Orb & Vallée de l'Hérault & Vallée de la Durance & PACA \\
\hline Khi $^{2}$ & 19,620 & 16,784 & 2,413 & 0,014 \\
\hline$\%$ inertie & 35,67 & 30,51 & 4,39 & 0,03 \\
\hline Test de Fischer & $>$ & $>$ & $<$ & $<$ \\
\hline Effectifs & 5 & 30 & 1 & 8 \\
\hline
\end{tabular}

Tableau 7 : Résultats pour le test de dépendance de la classe 4 à la variable de classification par zone de production Table 7: Dependency ratio between the group 4 and the classification by area of production.

Dans la seconde classification des échantillons, la première sous-classe dans laquelle se range l'échantillon AMG 804 de Carthagène se détache nettement des autres par l'absence d'échantillons de Saint-Pargoire. Elle est principalement caractérisée par la présence d'échantillons de l'atelier de Fréjus, Forum Julii (région PACA).

Malgré les résultats du test de Fisher, cette sous-classe contient trop peu d'échantillons pour être réellement représentative d'un groupe de production. Même si l'on compte les productions de Vendres et de Volonne comme des out-

\begin{tabular}{|l|c|c|c|}
\hline $\begin{array}{l}\text { Classe } 4 \\
\text { Sous-classe } 1\end{array}$ & Vendres & Volonnes & Fréjus \\
\hline Khi $^{2}$ & 1,389 & 3,803 & 15,211 \\
\hline \% inertie & 5,25 & 14,38 & 57,51 \\
\hline Test de Fischer & $>$ & $>$ & $>$ \\
\hline Effectifs & 2 & 1 & 4 \\
\hline
\end{tabular}

Tableau 8 : Résultats des tests de dépendance de la sous-classe 1 de la classe 4 à la variable de classification par atelier de production. Table 8: Dependency ratio between the sub-group 1 of group 4 and the classification by area of production. liers, on ne peut pas considérer que la représentation de l'atelier de Fréjus soit suffisante pour y lier l'individu AMG 804.

\section{- Classe 5}

Cette classe est principalement caractérisée par des valeurs fortes en silice, zirconium, cérium et dans une moindre mesure, en aluminium, potassium et titane. Les valeurs pour le fer et les éléments traces sidérophiles sont particulièrement faibles. Le nuage des points assez dispersé reflète une variance intra-classe élevée et une certaine hétérogénéité des signaux géochimiques. Malgré cette dispersion, les échantillons de cette classe se distinguent nettement des autres groupes de références dans l'espace factoriel.

Les échantillons de références de cette classe proviennent exclusivement de la région PACA. Trois échantillons proviennent de l'atelier de Toulon, trois de l'atelier de la Crau d'Hyères et les autres proviennent tous des ateliers de Fréjus. Deux échantillons de Carthagène (AMG 801 et AMG 802) sont présents dans cette classe. Le lien avec les ateliers de Fréjus semble concret. 


\begin{tabular}{|l|c|}
\hline Classe 5 & PACA \\
\hline Khi $^{2}$ & 96,896 \\
\hline$\%$ inertie & 81,03 \\
\hline Test de Fischer & $>$ \\
\hline Effectifs & 28 \\
\hline
\end{tabular}

Tableau 9 : Résultats pour le test de dépendance de la classe 5 à la variable de classification par zone de production.

Table 9: Dependency ratio between the group 5 and the classification by area of production.

\section{Attributions des échantillons}

Pour chaque classe ou sous-classe, l'analyse des nuées dynamiques autorise le calcul des distances de chaque individu au barycentre de la classe en prenant en compte les quatre axes factoriels choisis. En analysant la distribution de ces distances, il est possible de qualifier la place des échantillons de Carthagène dans les classes 1 et 5 où se dessine une cohérence entre la classification statistique et les zones de productions.

\section{- Classe 1 , sous-classe 4}

Dans la distribution des distances des échantillons au barycentre de cette sous-classe, la plupart des échantillons se trouvent à proximité de la distribution normale théorique. Le test des valeurs extrêmes (test de Dixon) indique que seul l'échantillon de Saint-Gilles qui se trouve en queue de distribution a une probabilité (inférieure à $50 \%$ ) d'être un outlier. Les trois échantillons de Carthagène AMG 798, AMG 800 et AMG 803 sont proches du centre la distribution et ne peuvent donc pas être considérés comme des outliers. D'après l'analyse de la distribution, l'attribution de ces trois échantillons de Carthagène à la zone de production délimitée semble robuste (figure 8).

\section{- Classe 5}

Dans la distribution des distances des échantillons au barycentre de cette classe, la plupart des échantillons se trouvent à proximité de la distribution normale théorique. Le test des valeurs extrêmes indique que les trois échantillons de l'atelier de la Crau d'Hyères ont des valeurs extrêmes (probabilité supérieure à $50 \%$ ). Ces outliers sont retirés de l'analyse de la distance au barycentre. Les deux individus de Carthagène AMG 801 et AMG 802 s'intègrent dans la distribution des échantillons de Fréjus et de Toulon (figure 9). La qualité de leur attribution par l'analyse des ND n'est donc pas remise en cause.

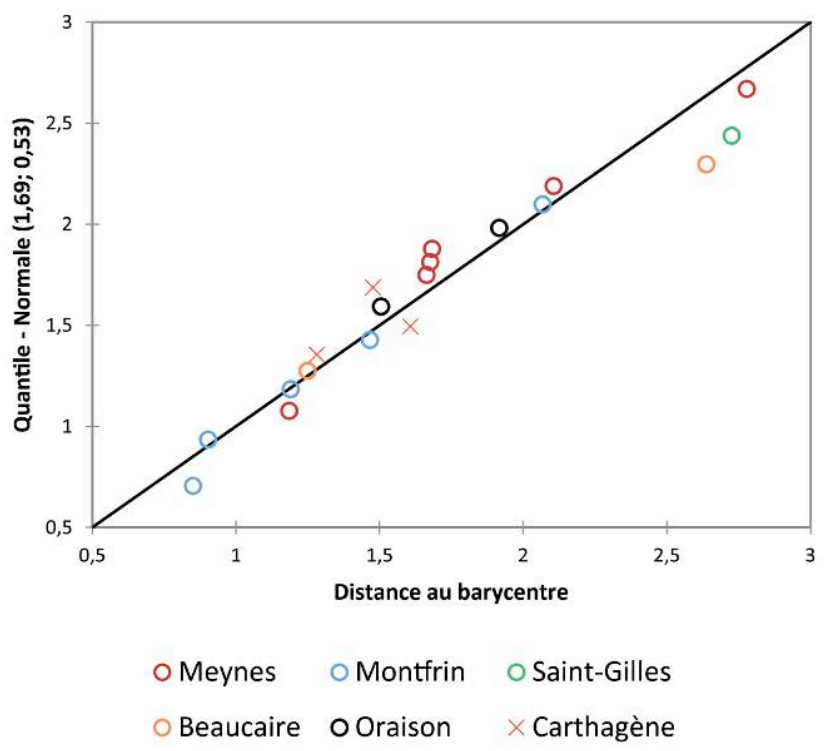

Figure 8 : (Voir planche couleur XX) Diagramme Q-Q des distances au barycentre de la sous-classe 4 de la classe 1.

Figure 8: (See colour plate XX) Q-Q plot of the distance from barycenter for the sub-group 4 in class 1 .

\section{Validation de la classification et probabilités d'attribution par l'analyse factorielle discriminante}

La première étape de la validation des attributions de l'analyse des N.D. consiste en la création d'un modèle différent qui, par comparaison, permet de valider le premier. Les individus de Carthagène en sont exclus afin que ceux-ci n'influencent pas sa construction.

Les résultats du test de Box (approximation asymptomatique du $\mathrm{Khi}^{2}$ ) et du test du Lambda de Wilks (approximation de Rao) (tableau 10) montrent la validité statistique du modèle : le test de Box rend compte de la covariance identique des groupes et le test du Lambda de Wilks valide l'égalité entre les $K$ centres de gravité. La règle de décision est bien linéaire et non quadratique.

Les résultats de la matrice de confusion pour la validation croisée montrent que les cinq classes réalisées en amont par la méthode des nuées dynamiques sont robustes et résistent à un second test statistique avec un taux de réussite sur la classification générale supérieur à $90 \%$.

La classification de la classe 1 est validée à plus de $88 \%$. Les attributions de sept échantillons de la vallée de l'Aude sont remisent en cause. La représentativité de la zone de production de la vallée du Gardon et de la Basse vallée du Rhône dans la sous-classe 4 n'est pas remise en cause. 


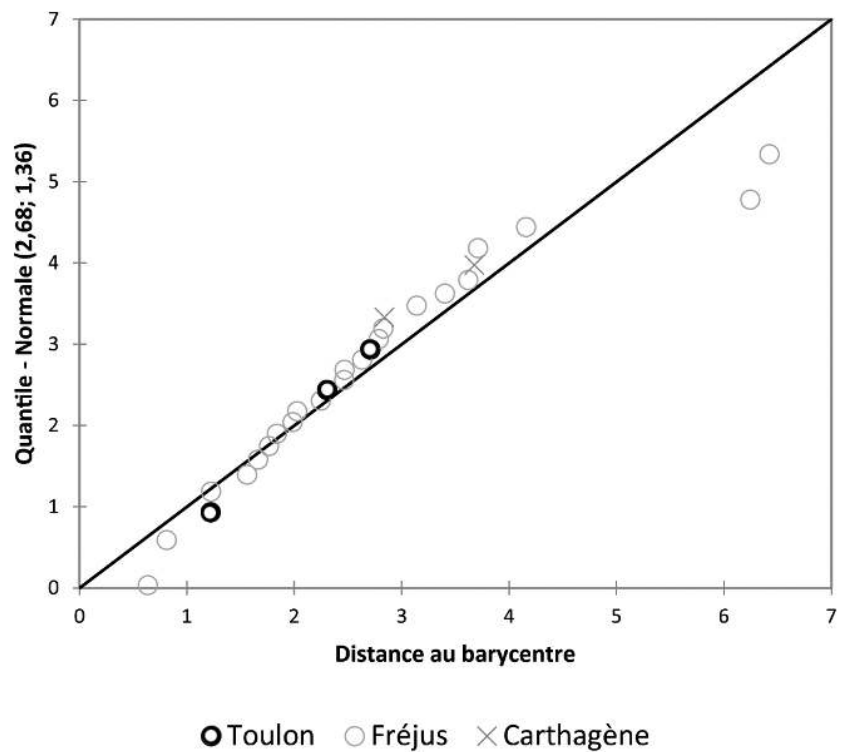

Figure 9 : Diagramme Q-Q des distances au barycentre de la classe 5 .

Figure 9: $Q-Q$ plot of the distance from barycenter for the group 5.

La classification de la classe 5 est validée à plus de $96 \%$ et confirme la bonne représentativité de la région PACA dans cette classe. Seul un échantillon de la Crau d'Hyères subit un changement d'affectation.

\begin{tabular}{|l|c|c|}
\hline & Test de Box & Test Lambda de Wilks \\
\hline -2Log(M) / Lambda & 453,635 & 0,020 \\
\hline F (Valeur observée) & 7,209 & 94,499 \\
\hline F (Valeur critique) & 1,318 & 1,583 \\
\hline p-value & $<0,0001$ & $<0,0001$ \\
\hline alpha & 0,05 & 0,05 \\
\hline
\end{tabular}

Tableau 10 : Résultats des tests de Box (approximation asymptomatique du $\mathrm{khi}^{2}$ ) et du test du Lambda de Wilks (approximation de Rao)

Table 10: Results of the Box test and Lambda's Wilks test.

\section{Prédiction statistique pour l'attribution des individus de Carthagène}

L'approche prédictive repose sur la règle d'affectation bayésienne. Les résultats sont présentés sous formes de probabilités a posteriori qui prennent en compte les probabilités a priori à travers la formule de Bayes. L'individu est attribué au groupe qui maximise cette probabilité. Il est cependant important d'observer les valeurs des résultats afin de vérifier la qualité de l'affectation. Le tableau des résultats pour la prédiction de classification des individus de Carthagène correspond aux résultats de la classification a priori réalisée par la méthode des nuées dynamiques.

\begin{tabular}{|l|c|c|c|}
\hline Individus & Affection a priori (N.D.) & Affection a posteriori (AFD) & Prédiction \\
\hline AMG 798 & Classe 1 - Sous classe 4 & Classe 1 - Sous classe 4 & $97,26 \%$ \\
\hline AMG 800 & Classe 1 - Sous classe 4 & Classe 1 - Sous classe 4 & $97,28 \%$ \\
\hline AMG 801 & Classe 5 & Classe 5 & $100,00 \%$ \\
\hline AMG 802 & Classe 5 & Classe 5 & $99,99 \%$ \\
\hline AMG 803 & Classe 1 - Sous classe 4 & Classe 1 - Sous classe 4 & $98,20 \%$ \\
\hline
\end{tabular}

Tableau 12 Résultats des probabilités d'attribution par la validation croisée pour les cinq échantillons de Carthagène attribués par l'analyse des ND.

Table 12: DFA cross-validation probability of assignment for the 5 samples assigned by dynamic cloud clustering.

\begin{tabular}{|l|c|c|c|c|c|c|c|}
\hline de \Vers & 1 & 2 & 3 & 4 & 5 & Total & $\%$ correct \\
\hline 1 & 55 & 3 & 4 & 0 & 0 & 62 & $88,71 \%$ \\
\hline 2 & 0 & 54 & 2 & 0 & 0 & 56 & $96,43 \%$ \\
\hline 3 & 1 & 0 & 67 & 0 & 0 & 68 & $98,53 \%$ \\
\hline 4 & 0 & 0 & 0 & 42 & 3 & 45 & $93,33 \%$ \\
\hline 5 & 0 & 1 & 0 & 0 & 29 & 30 & $96,67 \%$ \\
\hline Total & 56 & 58 & 73 & 42 & 32 & 261 & $94,64 \%$ \\
\hline
\end{tabular}

Tableau 11 : Matrice de validation croisée pour les cinq classes de l'analyse des ND.

Table 11: DFA cross-validation matrix for the 5 groups from the dynamic cloud clustering. 
Les échantillons AMG 799, AMG 800 et AMG 803 sont attribués à la sous classe 4 de classe 1 avec des valeurs de probabilités supérieures à $90 \%$.

Les échantillons AMG 801 et 802 sont attribués à la classe 5 avec une probabilité supérieure à $99 \%$. Cette classe étant représentative des échantillons de la zone de production de Fréjus et Toulon, ces deux échantillons de Carthagène peuvent être attribués à celle-ci.

Les échantillons AMG 799, AMG 804 et AMG 805 étant distribués dans des classes dont l'origine des échantillons est très hétérogène, il est impossible de les attribuer à une zone de production précise.

\section{Conclusion}

Les difficultés pour réaliser une classification statistique sur la base des résultats géochimiques, en cohérence avec les données archéologiques, sont réelles, comme nous l'avons indiqué. Cependant les résultats obtenus, en combinant plusieurs types d'outils complémentaires, sont positifs. Nous avons pu attribuer une provenance à six échantillons sur huit. Deux d'entre eux, AMG 801 de la villa Portmán et 802 de la domus de la Fortuna à Carthagène, proviennent de Fréjus. Trois autres, AMG 798 et AMG 800, de la villa Portmán, et AMG 800 de 803 de la domus de la Fortuna à Carthagène, ont pour origine la région Provence, Côte d'Azur. Les trois derniers, AMG 799 de la villa Portmán, AMG 804 de la domus de la Fortuna et AMG 805 de la rue Serreta à Carthagène, ne sont pas attribués. S'ils viennent de la même région, ils appartiennent à un groupe de production qu'il n’a pas été possible de reconnaître avec la méthode appliquée.

Il sera important, par ailleurs, pour être tout à fait au clair, de travailler sur les éventuelles productions locales de la zone de Carthagène et de comparer leurs argiles à celles de la Narbonnaise. On connaît déjà, en effet, une production d'amphores Gauloise 4, dans une zone voisine, à Dénia, plus au nord sur la côte (Laubenheimer, Gisbert Santonja 2001).

Ainsi, comme le laissaient penser les observations macroscopiques, et la typologie, la diffusion des amphores Gauloise 4 de Narbonnaise touche la région de Carthagène dans le sud-est de la Péninsule ibérique. Ces importations sont bien datées par les contextes archéologiques entre la fin du $\mathrm{II}^{\mathrm{e}}$ siècle et le début du $\mathrm{III}^{\mathrm{e}}$ siècle. Leur présence dans la région n'est peut-être pas si rare comme le montre la découverte d'un grand nombre d'exemplaires inédits dans le dragage du port d'Águilas, voisin de Carthagène. On notera, par ailleurs, que les sigillées sud-gauloises importées à Carthagène et dans la région depuis le $\mathrm{I}^{\mathrm{er}} \mathrm{s}$. de notre ère étaient des marchandises qui accompagnaient le vin sud gaulois.

La voie vers de nouvelles recherches est largement ouverte, tandis que les liaisons entre les exportations de vin de Narbonnaise et cette zone de la Péninsule semblent se confirmer.

\section{Remerciements}

Merci à Anne-Lise Binois et Alain Giosa, UMR 7041 ArScAn, pour leur relecture enrichissante et leurs commentaires.

\section{Bibliographie}

Aitchison J., 1986. The statistical analysis of compositional data, London: Chapman and Hall.

Aranegui C., Hassini H., 2010. Lixus-3, Saguntum Extra 8, Valence.

Baxter M.J., Beardah C.C., Freestone I.C., 2005. Compositional analysis of archaeological glasses'. In CODAWORK'05. Eds. G. Mateu i Figueras and C Barceló i Vidal. Girona, La Universitat, 2005, [http://hdl.handle. net/10256/688].

Bernau C., Riester M., Boulesteix A.L., Parmigiani G., Huttenhower C., Waldron L., Trippa L., 2014. Crossstudy validation for the assessment of prediction algorithms, Bioinformatics 30 (12), p. 105-112.

Boutsidis C., Mahoney M.W., Drineas P., 2009. Unsupervised Feature Selection for the Kmeans Clustering Problem. In Annual Advances in Neural Information Processing Systems, Proceedings of the NIPS'09 Conference.

Cattell R.B., 1966. The scree-test for the number of factors. Multivar. Behav. Res., 1, p. 245-276.

Diday E., 1971. Une nouvelle méthode en classification automatique et reconnaissance des formes la méthode des nuées dynamiques. Revue de Statistique Appliquée, 19.2, p. 19-33.

Fillipe V., 2013. 50 anos depois: as ânforas romanas da Praça da Figuera (Lisboa). In Arqueologia em Portugal, 150 anos, Associação dos Arqueologós Portugueses, Lisboa, p. 737-745.

Garcia Vargas E., 2012. Hispalis, (Sevilla, España) y el comercio mediterráneo en el alto Imperio. El testimonio de las ánforas, in Keay S. (ed.), Portus and the Mediterranean, Archaeological Monographs of the British School, Rome, p. 245-266.

Herut B., Sandler A., 2006. Normalization methods for pollutants in marine sediments: review and recommendations for the Mediterranean Israel Oceanographic and Limnological Research, IOLR Report, p. 1-23. 
KAISER H.F., 1960. The application of electronic computers to factor analysis. Educational and Psychological Measurement, 20, p. 141-151.

Kovarovic K., Aiello L.C., Cardini A., Lockwood C.A., 2011. Discriminant Function Analyses In Archaeology: Are Classification Rates Too Good To Be True?, Journal of Archaeological Science 38(11), p. 3006-3018.

LAUBENHEIMER F., à paraître. Le vin gaulois du Midi aux frontières de l'Empire romain et au-delà.

Laubenheimer F., 2015. Boire en Gaule, Paris, CNRS.

Laubenheimer F., Gisbert Santonja J. A., 2001. La standardisation des amphores Gauloise 4, des ateliers de Narbonnaise à la production de Dénia (Espagne). In F. Laubenheimer (dir.), 20 ans de recherches à Sallèles d'Aude, Presses Universitaires Franc-Comtoises.

Laubenheimer F., Schmitt A., 2009. Amphores vinaires de Narbonnaise. Production et grand commerce, Travaux de la Maison de l'Orient et de la Méditerranée, n 51, Lyon.
Lebart L., Piron M., Morineau A., 2006. Statistiques exploratoire multidimensionnelles: Visualisations et inférences en fouille de données, Paris, Dunod.

Panella C., 1973. Anfore. In Carandini A., Panella C. (éds), Le terme del Nuotatore, Ostia III, Studi Miscellanei 21, Rome.

Papin A., 2001. Étude expérimentale et spectroscopique de la cristallochimie du manganèse dans les silicates hydroxylés. Thèse de Doctorant, Université Orléans, 205 p.

Quevedo A., 2015. Contextos cerámicos y transformaciones urbanas en Carthago Nova (s. II-III d.C.), Roman and Late Antique Mediterranean Pottery, 7, Oxford (sous presse).

Tan P.N., Steinbach M., Kumar V., 2006. Introduction to Data Mining, Chapter 8, Cluster Analysis Basic Concepts and Algorithms, p. 125-146, Pearson Addison Wesley.

Wong M. A., 1982. A hybrid clustering method for identifying high-density clusters. Journal of the American Statistical Association, 77, p. 841-847. 


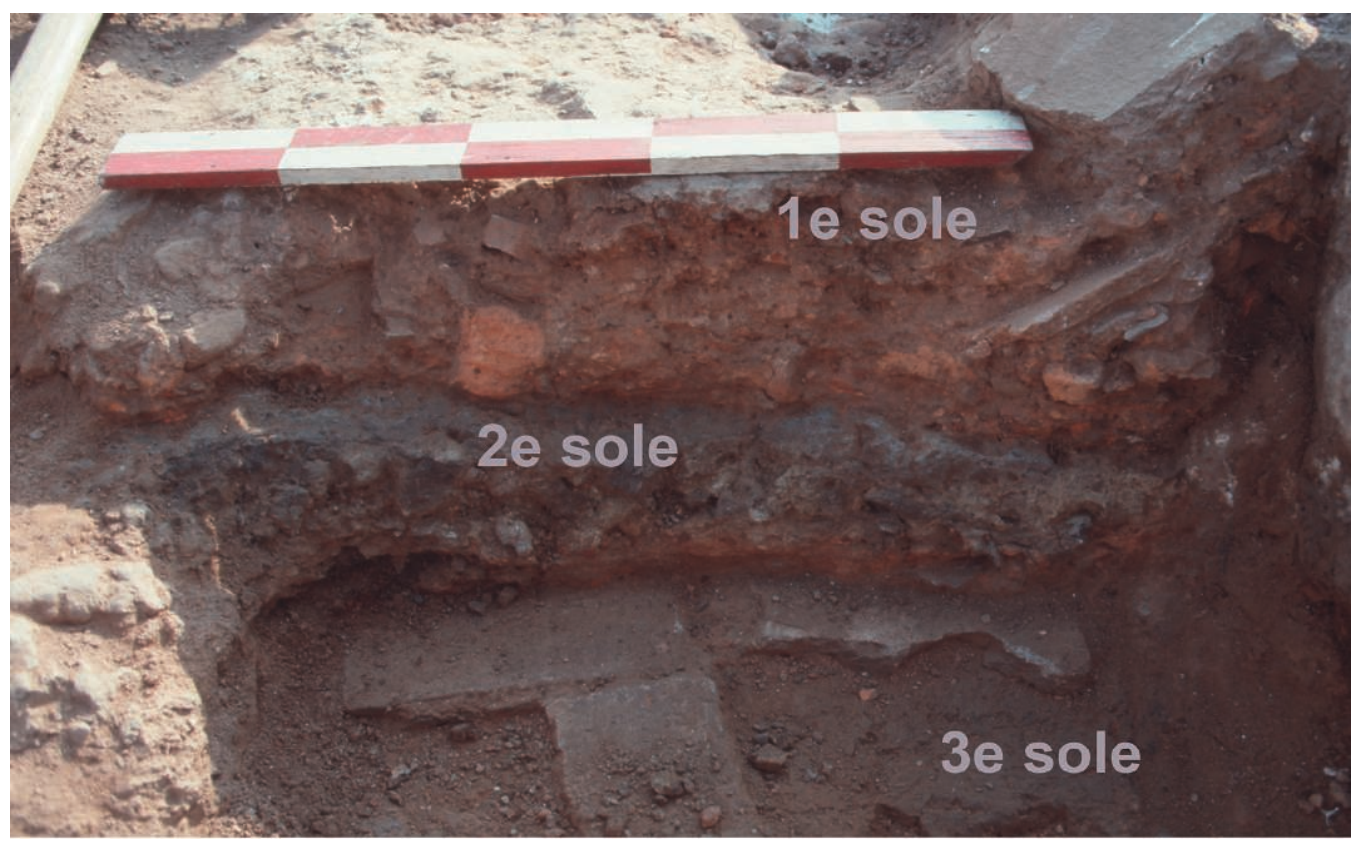

Figure 4 : Bruno Fajal et Dominique Marguerie, L'atelier de potier médiéval de la Picaudière (La Haute-Chapelle, Orne) : four, soles et combustible (p. 180)

Figure 7 : Thomas Delbey et al., Amphores vinaires Gauloise 4 de Narbonnaise (France) exportées à Carthagène (Espagne) ? Analyse statistique exploratoire multidimensionnelle de données géochimiques (p. 194)

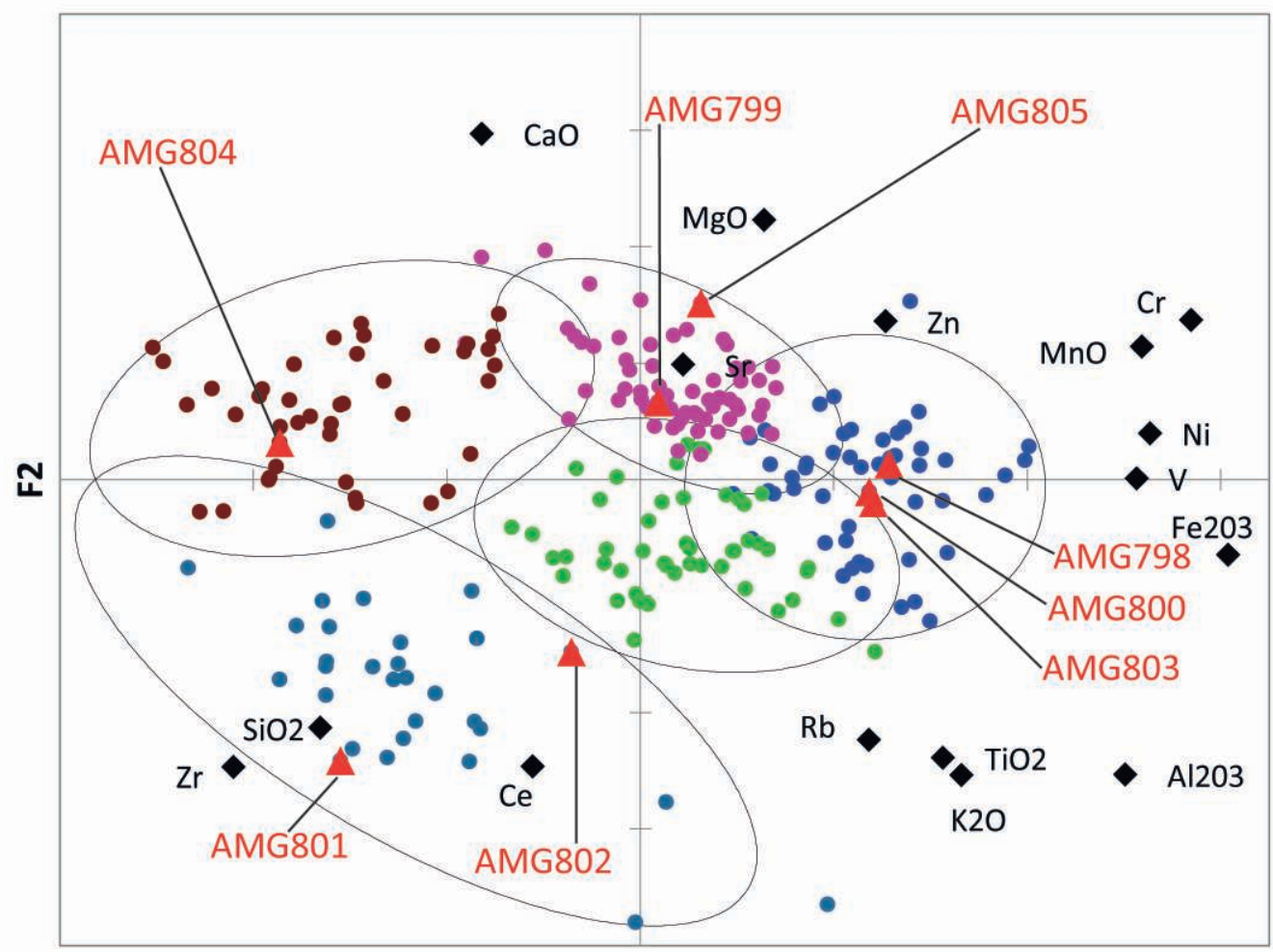

F1

- Classe $1 \bullet$ Classe $2 \bullet$ Classe $3 \bullet$ Classe $4 \bullet$ Classe $5 \Delta$ Echantillons Carthagène 
Figure 8 : Thomas Delbey et al., Amphores vinaires Gauloise 4 de Narbonnaise (France) exportées à Carthagène (Espagne) ? Analyse statistique exploratoire multidimensionnelle de données géochimiques (p. 196)

Figure 1 : Tony Rey et al., La cité médiévale d'AiguesMortes (Gard) : nouvelles données géomorphologiques et archéologiques sur un site méconnu (p. 204)

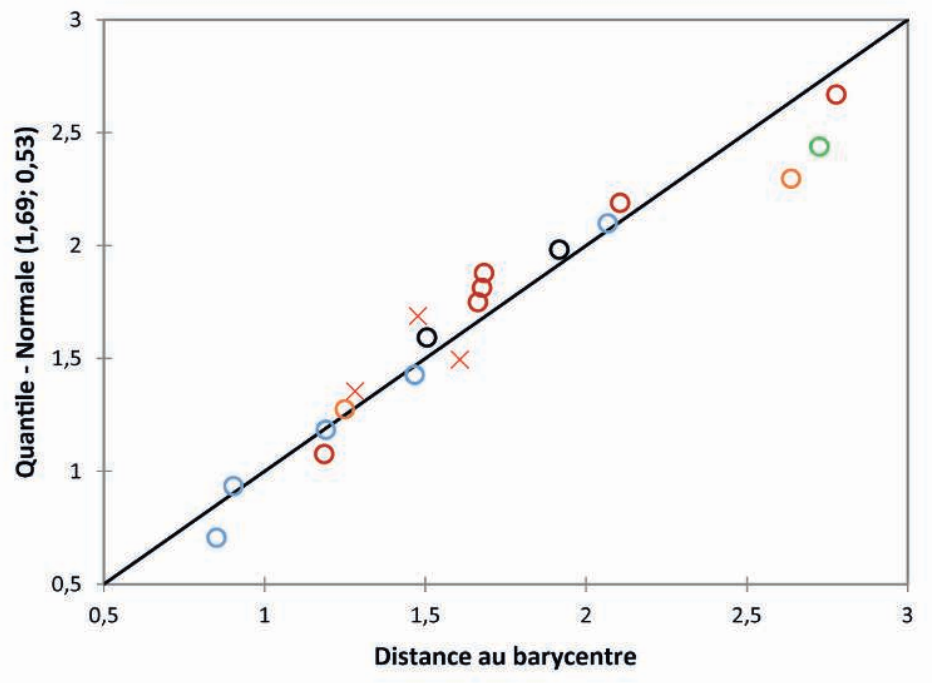

$\begin{array}{ll}\text { OMeynes OMontfrin } & \text { Saint-Gilles } \\ \text { OBeaucaire OOraison } \times \text { Carthagène }\end{array}$

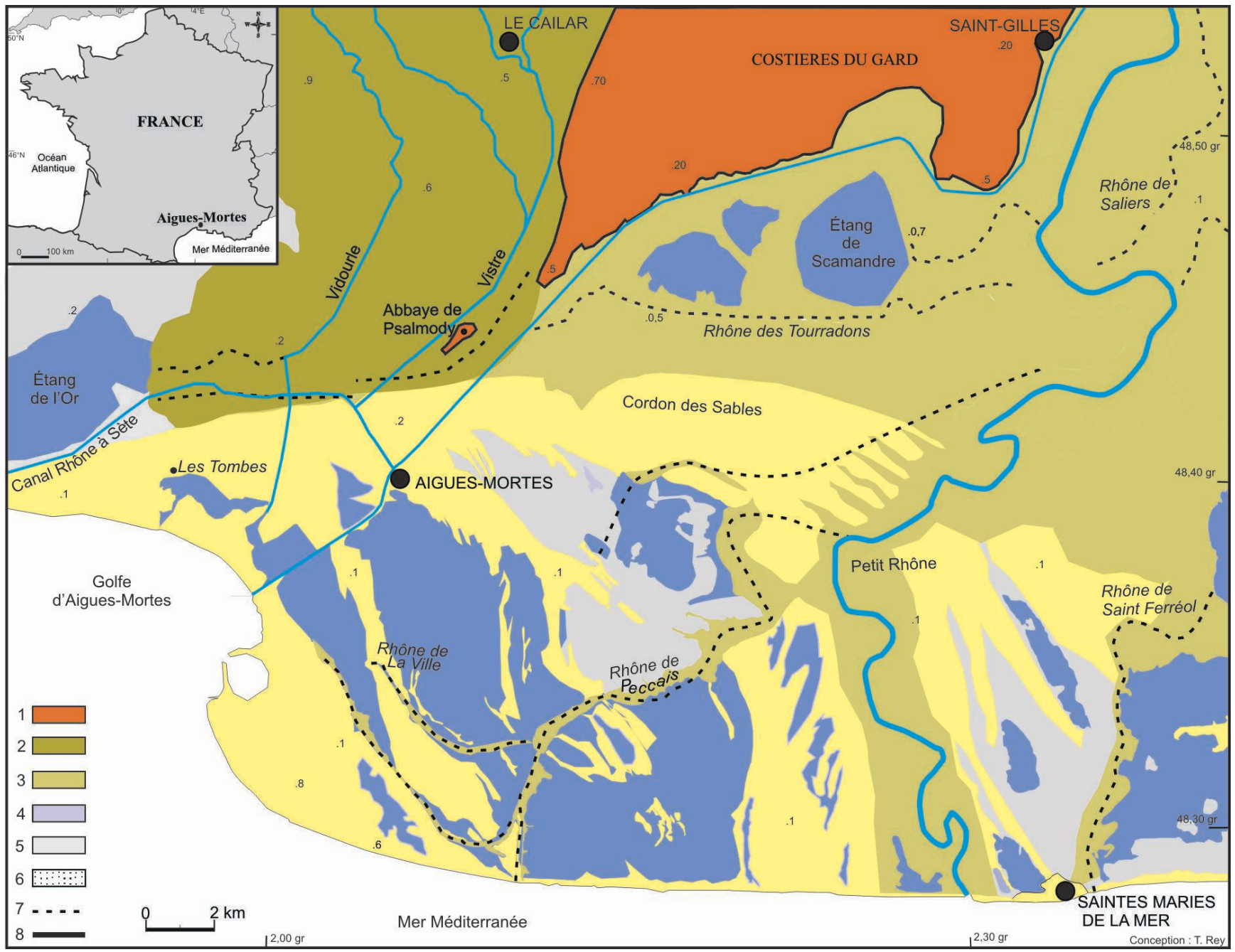

